\title{
Isolated singularities for the $n$-Liouville equation
}

\author{
Pierpaolo Esposito ${ }^{1}$
}

Received: 21 August 2020 / Accepted: 13 May 2021 / Published online: 29 June 2021

(c) The Author(s) 2021

\begin{abstract}
In dimension $n$ isolated singularities - at a finite point or at infinity - for solutions of finite total mass to the $n$-Liouville equation are of logarithmic type. As a consequence, we simplify the classification argument in Esposito (Anal Non Linéaire 35(3):781-801, 2018) and establish a quantization result for entire solutions of the singular $n$-Liouville equation.
\end{abstract}

Mathematics Subject Classification 35B08 $\cdot 35 \mathrm{~B} 33 \cdot 35 \mathrm{~J} 92 \cdot 35 \mathrm{C} 05$

\section{Introduction}

The behavior near an isolated singularity has been discussed by Serrin in [19,20] for a very general class of second-order quasi-linear equations. The simplest example is given by the prototypical equation $-\Delta_{n} u=f$, where $\Delta_{n}(\cdot)=\operatorname{div}\left(|\nabla(\cdot)|^{n-2} \nabla(\cdot)\right), n \geq 2$, is the $n$ Laplace operator. In dimension $n$, the case $f \in L^{1}$ is very delicate as it represents a limiting situation where Serrin's results do not apply. We will be interested in the $n$-Liouville equation, where $f$ is taken as an exponential function of $u$ according to Liouville's seminal paper [16], and the singularity might be at a finite point or at infinity.

To this aim, it is enough to consider the generalized $n$-Liouville equation

$$
-\Delta_{n} u=|x|^{n \alpha} e^{u} \text { in } \Omega \backslash\{0\}, \int_{\Omega}|x|^{n \alpha} e^{u}<+\infty
$$

on an open set $\Omega \subset \mathbb{R}^{n}$ with $0 \in \Omega$, and we will be concerned with describing the behavior of $u$ at 0 . A solution $u$ of (1.1) stands for a function $u \in C_{l o c}^{1, \eta}(\Omega \backslash\{0\})$ which satisfies

$$
\int_{\Omega}|\nabla u|^{n-2}\langle\nabla u, \nabla \varphi\rangle=\int_{\Omega}|x|^{n \alpha} e^{u} \varphi \quad \forall \varphi \in C_{0}^{1}(\Omega \backslash\{0\}) .
$$

Communicated by A. Malchiodi.

Partially supported by Gruppo Nazionale per l'Analisi Matematica, la Probabilitá e le loro Applicazioni (GNAMPA) of the Istituto Nazionale di Alta Matematica (INdAM).

Pierpaolo Esposito

esposito@mat.uniroma3.it

1 Dipartimento di Matematica e Fisica, Università degli Studi Roma Tre, Largo S. Leonardo Murialdo 1, 00146 Roma, Italy 
The regularity assumption on $u$ is not restrictive since a solution in $W_{\text {loc }}^{1, n}(\Omega \backslash\{0\})$ is automatically in $C_{l o c}^{1, \eta}(\Omega \backslash\{0\})$, for some $\eta \in(0,1)$, thanks to [9,19,22], see Theorem 2.3 in [11].

Concerning the behavior near an isolated singularity, our main result is

Theorem 1.1 Let $u$ be a solution of (1.1). Then there exists $\gamma>-n^{n}|\alpha+1|^{n-2}(\alpha+1) \omega_{n}$, $\omega_{n}=\left|B_{1}(0)\right|$, so that

$$
-\Delta_{n} u=|x|^{n \alpha} e^{u}-\gamma \delta_{0} \text { in } \Omega
$$

with

$$
u-\gamma\left(n \omega_{n}|\gamma|^{n-2}\right)^{-\frac{1}{n-1}} \log |x| \in L_{\mathrm{loc}}^{\infty}(\Omega)
$$

and

$$
\lim _{x \rightarrow 0}\left[|x| \nabla u(x)-\gamma\left(n \omega_{n}|\gamma|^{n-2}\right)^{-\frac{1}{n-1}} \frac{x}{|x|}\right]=0 .
$$

The case $\alpha=-2$ is relevant for the asymptotic behavior at infinity for solutions $u$ of

$$
-\Delta_{n} u=e^{u} \text { in } \Omega, \int_{\Omega} e^{u}<+\infty,
$$

where $\Omega$ is an unbounded open set so that $B_{R}(0)^{c} \subset \Omega$ for some $R>0$. Indeed, let us recall that $\Delta_{n}$ is invariant under Kelvin transform: if $u$ solves $(1.1)$, then $\hat{u}(x)=u\left(\frac{x}{|x|^{2}}\right)$ does satisfy

$$
-\Delta_{n} \hat{u}=|x|^{-2 n}\left(-\Delta_{n} u\right)\left(\frac{x}{|x|^{2}}\right)=|x|^{-n(\alpha+2)} e^{\hat{u}} \text { in } \hat{\Omega}=\left\{x \neq 0: \frac{x}{|x|^{2}} \in \Omega\right\} .
$$

By Theorem 1.1 applied with $\alpha=-2$ to $\hat{u}$ at 0 we find:

Corollary 1.2 Let $u$ be a solution of (1.5) on an unbounded open set $\Omega$ with $B_{R}(0)^{c} \subset \Omega$ for some $R>0$. Then there holds

$$
u=-\left(\frac{\gamma_{\infty}}{n \omega_{n}}\right)^{\frac{1}{n-1}} \log |x|+O(1)
$$

as $|x| \rightarrow \infty$ for some $\gamma_{\infty}>n^{n} \omega_{n}$. In particular, when $\Omega=\mathbb{R}^{n}$ there holds

$$
u=-\left(\frac{1}{n \omega_{n}} \int_{\mathbb{R}^{n}} e^{u}\right)^{\frac{1}{n-1}} \log |x|+O(1)
$$

as $|x| \rightarrow \infty$.

When $n=2$ the asymptotic expansion (1.8) is a well known property established in [6] by means of the Green representation formula — unfortunately not available in the quasi-linear case-and of the growth properties of entire harmonic functions. Notice that

$$
\gamma_{\infty}=\int_{\mathbb{R}^{n}}|x|^{-2 n} e^{\hat{u}}=\int_{\mathbb{R}^{n}} e^{u}
$$

follows by integrating (1.2) written for $\hat{u}$ on $\mathbb{R}^{n}$. Property (1.8) has been already proved in [11] under the assumption $\gamma_{\infty}>n^{n} \omega_{n}$ and the present full generality allows to simplify the classification argument in [11]: a Pohozaev identity leads for $\gamma_{\infty}$ to the quantization property

$$
\int_{\mathbb{R}^{n}} e^{u}=n\left(\frac{n^{2}}{n-1}\right)^{n-1} \omega_{n}
$$


and an isoperimetric argument concludes the classification result thanks to (1.9).

In the punctured plane $\Omega=\mathbb{R}^{n} \backslash\{0\}$ the isoperimetric argument fails and in general the classification result is no longer available. The two-dimensional case $n=2$ has been treated via complex analysis in [7,17]: solutions $u$ to

$$
-\Delta u=e^{u}-\gamma \delta_{0} \text { in } \mathbb{R}^{2}, \int_{\mathbb{R}^{2}} e^{u}<+\infty,
$$

have been classified for $\gamma>-4 \pi$ of the form

$$
u(x)=\log \frac{8(\alpha+1)^{2} \lambda^{2}|x|^{2 \alpha}}{\left(1+\lambda^{2}\left|x^{\alpha+1}+c\right|^{2}\right)^{2}}, \alpha=\frac{\gamma}{4 \pi},
$$

with $\lambda>0$ and a complex number $c=0$ if $\alpha \notin \mathbb{N}$, and in particular satisfy

$$
\int_{\mathbb{R}^{n}} e^{u}=8 \pi(\alpha+1) .
$$

The structure of entire solutions $u$ to (1.10) changes drastically passing from radial solutions when $\alpha \notin \mathbb{N}$ to non-radial solutions when $\alpha \in \mathbb{N}$ (and $c \neq 0$ ). Unfortunately a PDE approach is not available for $n=2$ and a classification result is completely out of reach when $n \geq 3$. However, quantization properties are still in order as it follows by Theorem 1.1 and the Pohozaev identities:

Theorem 1.3 Let $u$ be a solution of

$$
-\Delta_{n} u=e^{u}-\gamma \delta_{0} \text { in } \mathbb{R}^{n}, \int_{\mathbb{R}^{n}} e^{u}<+\infty .
$$

Then $\gamma>-n^{n} \omega_{n}$ and

$$
\int_{\mathbb{R}^{n}} e^{u}=\gamma+\gamma_{\infty}
$$

with $\gamma_{\infty}$ the unique solution in $\left(n^{n} \omega_{n},+\infty\right)$ of

$$
\frac{n-1}{n}\left(n \omega_{n}\right)^{-\frac{1}{n-1}} \gamma_{\infty}^{\frac{n}{n-1}}-n \gamma_{\infty}=n \gamma+\frac{n-1}{n}\left(n \omega_{n}\right)^{-\frac{1}{n-1}}|\gamma|^{\frac{n}{n-1}} .
$$

When $n=2$ notice that for $\gamma>-4 \pi$ the unique solution $\gamma_{\infty}>4 \pi$ of (1.14) is given explicitly as $\gamma_{\infty}=\gamma+8 \pi$ and then $\int_{\mathbb{R}^{n}} e^{u}=2 \gamma+8 \pi=8 \pi(\alpha+1)$ in accordance with (1.11). To have Theorem 1.3 meaningful, in Sect. 4 we will show the existence of a family of radial solutions $u$ to (1.12) but we don't know whether other solutions might exist or not, depending on the value of $\gamma$. Notice that (1.10) is equivalent to

$$
-\Delta v=|x|^{2 \alpha} e^{v} \text { in } \mathbb{R}^{2}, \int_{\mathbb{R}^{2}}|x|^{2 \alpha} e^{v}<+\infty,
$$

in terms of $v=u-2 \alpha \log |x|$. For $n \geq 3$ such equivalence breaks down and the problem

$$
-\Delta_{n} v=|x|^{n \alpha} e^{v} \text { in } \mathbb{R}^{n}, \int_{\mathbb{R}^{n}}|x|^{n \alpha} e^{v}<+\infty,
$$

has its own interest, independently of (1.12). As in Theorem 1.3, for (1.15) we have the following quantization result: 
Theorem 1.4 Let $v$ be a solution of (1.15). Then $\alpha>-1$ and

$$
\int_{\mathbb{R}^{n}}|x|^{n \alpha} e^{v}=n\left(\frac{n^{2}}{n-1}\right)^{n-1}(\alpha+1)^{n-1} \omega_{n} .
$$

Radial solutions $v$ of (1.15) can be easily obtained as $v=n \log (\alpha+1)+u\left(|x|^{\alpha+1}\right)$ in terms of a radial entire solution $u$ to (1.5). Thanks to the classification result in [11], for (1.15) we can therefore exhibit the following family of radial solutions:

$$
v_{\lambda}=\log \frac{c_{n}(\alpha+1)^{n} \lambda^{n}}{\left(1+\lambda^{\frac{n}{n-1}}|x|^{\frac{n(\alpha+1)}{n-1}}\right)^{n}}, c_{n}=n\left(\frac{n^{2}}{n-1}\right)^{n-1} .
$$

Problems with exponential nonlinearities on a bounded domain with given singular sources can exhibit non-compact solution-sequences, whose shape near a blow-up point is asymptotically described by the limiting problem (1.12). In the regular case (i.e. in absence of singular sources) a concentration-compactness principle has been established [5] for $n=2$ and [1] for $n \geq 2$. In the non-compact situation the exponential nonlinearity concentrates at the blow-up points as a sum of Dirac measures. Theorem 1.3 gives information on the concentration mass of such Dirac measures at a singular blow-up point, which is expected bo te a super-position of several masses $c_{n} \omega_{n}$ carried by multiple sharp collapsing peaks governed by (1.12) $\gamma=0$ and possibly the mass (1.13) of a sharp peak described by (1.12). In the regular case such quantization property on the concentration masses has been proved [14] for $n=2$ and extended [12] to $n \geq 2$ by requiring an additional boundary assumption, while the singular case has been addressed in [2,21] for $n=2$. For Theorem 1.4 a similar comment is in order.

Let us briefly explain the main ideas behind Theorem 1.1. We can re-adapt the argument in [11] to show that $u \in \bigcap_{1 \leq q<n} W_{\text {loc }}^{1, q}(\Omega)$ and then $u$ satisfies (1.2) for some $\gamma \in \mathbb{R}$. On a radial ball $B \subset \subset \Omega$ decompose $u$ as $u=u_{0}+h$, where $h$ is given by

$$
\Delta_{n} h=\gamma \delta_{0} \text { in } B, \quad h=u \text { on } \partial B
$$

and satisfies $(1.3)$ thanks to $[13,19,20]$. The key property stems from a simple observation: $|x|^{n \alpha} e^{h} \in L^{1}$ near 0 implies $|x|^{n \alpha} e^{h} \in L^{p}$ near 0 for some $p>1$ whenever $h$ has a logarithmic singularity at 0 . Back to $[19,20]$ thanks to such improved integrability, one aims to show that $u_{0} \in L^{\infty}(B)$ and then $u$ has the same logarithmic behavior (1.3) as $h$. In order to develop a regularity theory for the solution $u_{0}$ of

$$
-\Delta_{n}\left(u_{0}+h\right)+\Delta_{n} h=|x|^{n \alpha} e^{u_{0}+h} \text { in } B, u_{0}=0 \text { on } \partial B,
$$

the crucial point is to establish several integral inequalities involving $u_{0}$ paralleling the estimates available for entropy solutions in [1,3] and for $W^{1, n}$-solutions in [19]. To this aim, we make use of the deep uniqueness result [10] to show that $u$ can be regarded as a Solution Obtained as Limit of Approximations (the so-called SOLA, see for example [4]).

The paper is organized as follows. In Sect. 2 we develop the above argument to prove Theorem 1.1. Section 3 is devoted to establish Theorems 1.3-1.4 via Pohozaev identities: going back to an idea of Y.Y. Li and N. Wolanski for $n=2$, the Pohozaev identities have revealed to be a fundamental tool to derive information on the mass of a singularity (see for example $[2,12,18])$. In Sect. 4 a family of radial solutions $u$ to (1.12) is constructed. 


\section{Proof of Theorem 1.1}

Assume $B_{1}(0) \subset \subset \Omega$. Let us first establish the following property on $u$.

Proposition 2.1 Let $u$ be a solution of (1.1). There exists $C>0$ so that

$$
u(x) \leq C-n(\alpha+1) \log |x| \quad \text { in } B_{1}(0) \backslash\{0\} .
$$

Proof Letting $U_{r}(y)=\hat{u}\left(\frac{y}{r}\right)+n(\alpha+1) \log r=u\left(\frac{r y}{|y|^{2}}\right)+n(\alpha+1) \log r$ for $0<r \leq \frac{1}{2}$, we have that $U_{r}$ solves

$$
\begin{gathered}
-\Delta_{n} U_{r}=|y|^{-n(\alpha+2)} e^{U_{r}} \quad \text { in } \mathbb{R}^{n} \backslash B_{\frac{1}{2}}(0), \\
\int_{\mathbb{R}^{n} \backslash B_{\frac{1}{2}}(0)}|y|^{-n(\alpha+2)} e^{U_{r}}=\int_{B_{2 r}(0)}|x|^{n \alpha} e^{u}
\end{gathered}
$$

in view of (1.6). Given a ball $B_{\frac{1}{2}}\left(x_{0}\right)$ for $x_{0} \in \mathbb{S}^{n-1}$, let us consider the $n$-harmonic function $H_{r}$ in $B_{\frac{1}{2}}\left(x_{0}\right)$ so that $H_{r}=U_{r}$ on $\partial B_{\frac{1}{2}}\left(x_{0}\right)$. By the weak maximum principle we deduce that $H_{r} \leq U_{r}$ in $B_{\frac{1}{2}}\left(x_{0}\right)$ and then

$$
\begin{aligned}
\int_{B_{\frac{1}{2}}\left(x_{0}\right)}\left(H_{r}\right)_{+}^{n} & \leq \int_{B_{\frac{1}{2}}\left(x_{0}\right)}\left(U_{r}\right)_{+}^{n} \leq n ! \int_{B_{\frac{1}{2}}\left(x_{0}\right)} e^{U_{r}} \\
& \leq C \int_{\mathbb{R}^{n} \backslash B_{\frac{1}{2}}(0)}|y|^{-n(\alpha+2)} e^{U_{r}} \leq C \int_{\Omega}|x|^{n \alpha} e^{u}<+\infty
\end{aligned}
$$

for all $0<r \leq \frac{1}{2}$. By the estimates in [19] we have that there exists $C>0$ so that

$$
\left\|H_{r}\right\|_{\infty, B_{\frac{1}{4}}\left(x_{0}\right)} \leq C
$$

for all $0<r \leq \frac{1}{2}$. At the same time, by the exponential estimate in [1] we have that there exist $0<r_{0} \leq \frac{1}{2}$ and $C>0$ so that

$$
\int_{B_{\frac{1}{2}}\left(x_{0}\right)} e^{2\left|U_{r}-H_{r}\right|} \leq C
$$

for all $0<r \leq r_{0}$ in view of $\lim _{r \rightarrow 0^{+}} \int_{B_{\frac{1}{2}}\left(x_{0}\right)}|y|^{-n(\alpha+2)} e^{U_{r}}=0$ thanks to (1.1) and (2.2). Since $|y|^{-n(\alpha+2)} e^{U_{r}} \leq C e^{\left|U_{r}-H_{r}\right|}$ on $B_{\frac{1}{4}}\left(x_{0}\right)$ for all $0<r \leq \frac{1}{2}$ in view of (2.4), we deduce that $|y|^{-n(\alpha+2)} e^{U_{r}}$ and $\left(U_{r}\right)_{+}^{\frac{n}{2}}$ are uniformly bounded in $L^{2}\left(B_{\frac{1}{4}}\left(x_{0}\right)\right)$ for all $0<r \leq r_{0}$ in view of (2.3) and (2.5). Thanks again to the estimates in [19], we finally deduce that

$$
\left\|U_{r}^{+}\right\|_{\infty, B_{\frac{1}{8}}}\left(x_{0}\right) \leq C
$$

for all $0<r \leq r_{0}$. Since $\mathbb{S}^{n-1}$ can be covered by a finite number of balls $B_{\frac{1}{8}}\left(x_{0}\right), x_{0} \in \mathbb{S}^{n}$, going back to $u$ from (2.6) one deduces that

$$
u(x) \leq C-n(\alpha+1) \log |x|
$$

for all $|x|=r \leq r_{0}$. Since this estimate does hold in $B_{1}(0) \backslash B_{r_{0}}(0)$ too, we have established the validity of (2.1). 
From now on, set $B=B_{r}(0)$ for $0<r \leq 1$. We are now ready to establish the starting point for the argument we will develop in the sequel. There holds

Proposition 2.2 Let $u$ be a solution of (1.1). Then

$$
u \in \bigcap_{1 \leq q<n} W^{1, q}(\Omega) .
$$

Proof Let us go through the argument in [11] to obtain $W^{1, q}$-estimates on $u$. For $0<\epsilon<$ $r<1$ let us introduce $h_{\epsilon, r} \in W^{1, n}\left(A_{\epsilon, r}\right), A_{\epsilon, r}:=B \backslash \overline{B_{\epsilon}(0)}$, as the solution of

$$
\Delta_{n} h_{\epsilon, r}=0 \text { in } A_{\epsilon, r}, h_{\epsilon, r}=u \text { on } \partial A_{\epsilon, r} .
$$

Regularity issues for quasi-linear PDEs involving $\Delta_{n}$ are well established since the works of DiBenedetto, Evans, Lewis, Serrin, Tolksdorf, Uhlenbeck, Uraltseva. For example, by [9, $15,19,22]$ we deduce that $h_{\epsilon, r}, u_{\epsilon, r}=u-h_{\epsilon, r} \in C^{1, \eta}\left(\overline{A_{\epsilon, r}}\right)$ and $u_{\epsilon, r}$ satisfies

$$
-\Delta_{n}\left(u_{\epsilon, r}+h_{\epsilon, r}\right)+\Delta_{n} h_{\epsilon, r}=|x|^{n \alpha} e^{u} \text { in } A_{\epsilon, r}, u_{\epsilon, r}=0 \text { on } \partial A_{\epsilon, r} .
$$

By the techniques in $[1,3,4]$ we have the following estimates, see Proposition 2.1 in [11]: for all $1 \leq q<n$ and all $p \geq 1$ there exist $0<r_{0}<1$ and $C>0$ so that

$$
\int_{A_{\epsilon, r}}\left|\nabla u_{\epsilon, r}\right|^{q}+\int_{A_{\epsilon, r}} e^{p u_{\epsilon, r}} \leq C
$$

for all $0<\epsilon<r \leq r_{0}$ thanks to (2.8) and $\lim _{r \rightarrow 0^{+}} \int_{B}|x|^{n \alpha} e^{u}=0$. Since by the Sobolev embedding $W_{0}^{1, \frac{n}{2}}\left(B_{1}(0)\right) \hookrightarrow L^{n}\left(B_{1}(0)\right)$ there holds $\int_{A_{\epsilon, r}}\left|u_{\epsilon, r}\right|^{n} \leq C$ for all $0<\epsilon<r \leq r_{0}$ in view of (2.9) with $q=\frac{n}{2}$ and $A_{\epsilon, r} \subset B_{1}(0)$, we have that

$$
\left\|h_{\epsilon, r}\right\|_{L^{n}(A)} \leq C(A) \quad \forall A \subset \subset \bar{B} \backslash\{0\}, \forall 0<\epsilon<r \leq r_{0}
$$

in view of $u \in C_{l o c}^{1, \eta}\left(B_{1}(0) \backslash\{0\}\right)$ and then

$$
\left\|h_{\epsilon, r}\right\|_{C^{1, \eta}(A)} \leq C(A) \quad \forall A \subset \subset \bar{B} \backslash\{0\}, \forall 0<\epsilon<r \leq r_{0}
$$

thanks to $[9,15,19,22]$. By the Ascoli-Arzelá's Theorem and a diagonal process we can find a sequence $\epsilon \rightarrow 0$ so that $h_{\epsilon, r} \rightarrow h_{r}$ and $u_{\epsilon, r} \rightarrow u_{r}:=u-h_{r}$ in $C_{\text {loc }}^{1}(\bar{B} \backslash\{0\})$ as $\epsilon \rightarrow 0$, where $h_{r} \leq u$ is a $n$-harmonic function in $B \backslash\{0\}$ and $u_{r}$ satisfies

$$
u_{r} \in W_{0}^{1, q}(B), \quad e^{u_{r}} \in L^{p}(B)
$$

for all $1 \leq q<n$ and all $p \geq 1$ if $r$ is sufficiently small in view of (2.9). Since

$$
h_{r}(x) \leq C-n(\alpha+1) \log |x| \quad \text { in } B
$$

in view of $h_{r} \leq u$ and (2.1), we have that $H^{\lambda}(y)=-\frac{h_{r}(\lambda y)}{\log \lambda}$ is a $n$-harmonic function in $B_{\frac{r}{\lambda}}(0)$ so that $H^{\lambda} \leq n(\alpha+1)+1$ in $B_{2}(0) \backslash B_{\frac{1}{2}}(0)$ for all $0<\lambda \leq \lambda_{0}$, where $\lambda_{0} \in\left(0, \frac{r}{2}\right]$ is a suitable small number. By the Harnack inequality in Theorem 7- [19] applied to $n(\alpha+1)+1-H^{\lambda} \geq 0$ we deduce that

$$
\max _{|y|=1} H^{\lambda} \leq C\left[\frac{n|\alpha+1|+1}{C}+\min _{|y|=1} H^{\lambda}\right]
$$

for all $0<\lambda \leq \lambda_{0}$, for a suitable $C \in(0,1)$. There are two possibilities: 
- either $\min _{|y|=1} H^{\lambda} \geq-\frac{n|\alpha+1|+1}{C}$ for all $0<\lambda \leq \lambda_{1}$ and some $\lambda_{1} \in\left(0, \lambda_{0}\right]$, which implies $\max _{|y|=1}\left|H^{\lambda}\right| \leq \frac{n|\alpha+1|+1}{C}$ for all $0<\lambda \leq \lambda_{1}$ and in particular

$$
\left|h_{r}\right| \leq-C_{0} \log |x| \quad \text { in } B_{\lambda_{1}}(0)
$$

for some $C_{0}>0$;

- or $\min _{|y|=1} H^{\lambda_{n}} \leq-\frac{n|\alpha+1|+1}{C}$ for a sequence $\lambda_{n} \downarrow 0$, which implies $\max _{|y|=1} H^{\lambda_{n}} \leq 0$ in view of (2.11) and in turn $h_{r} \leq 0$ on $|x|=\lambda_{n}$ for all $n \in \mathbb{N}$, leading to

$$
h_{r} \leq 0 \quad \text { in } B_{\lambda_{1}}(0)
$$

by the weak maximum principle.

Notice that (2.13) implies the validity of (2.12) for some $C_{0}>0$ in view of Theorem 12- [19]. Thanks to (2.12) one can apply Theorem 1.1- [13] to show that

$$
h_{r} \in W^{1, q}(B)
$$

for all $1 \leq q<n$ and there exists $\gamma_{r} \in \mathbb{R}$ so that

$$
h_{r}-\gamma_{r}\left(n \omega_{n}\left|\gamma_{r}\right|^{n-2}\right)^{-\frac{1}{n-1}} \log |x| \in L^{\infty}(B), \quad \Delta_{n} h_{r}=\gamma_{r} \delta_{0} \text { in } B .
$$

In particular, $u \in \bigcap_{1 \leq q<n} W^{1, q}(\Omega)$ in view of (2.10) and (2.14), and (2.7) is established.

Even if $\gamma_{r}>-n^{n}|\alpha+1|^{n-2}(\alpha+1) \omega_{n}$, at this stage we cannot exclude that $\lim _{r \rightarrow 0} \gamma_{r}=$ $-n^{n}|\alpha+1|^{n-2}(\alpha+1) \omega_{n}$. Therefore, we are not able to use (2.10) and (2.15) for improving the exponential integrability on $u$ to reach $|x|^{n \alpha} e^{u}=|x|^{n \alpha} e^{h_{r}} e^{u_{r}} \in L^{p}$ near 0 for some $p>1$ and $r$ sufficiently small, as it would be necessary to prove $L^{\infty}$-bounds on $u_{r}$ via (2.8) on $u_{\epsilon, r}$.

We need to argue in a different way. Since $u \in W^{1, n-1}(\Omega)$ in view of (2.7), we can extend (1.1) at 0 as

$$
-\Delta_{n} u=|x|^{n \alpha} e^{u}-\gamma \delta_{0} \text { in } \Omega .
$$

To see it, let $\varphi \in C_{0}^{1}(\Omega)$ and consider a function $\chi_{\epsilon} \in C^{\infty}(\Omega)$ with $0 \leq \chi_{\epsilon} \leq 1, \chi_{\epsilon}=0$ in $B_{\frac{\epsilon}{2}}(0), \chi_{\epsilon}=1$ in $\Omega \backslash B_{\epsilon}(0)$ and $\epsilon\left|\nabla \chi_{\epsilon}\right| \leq C$. Taking $\chi_{\epsilon} \varphi \in C_{0}^{1}(\Omega \backslash\{0\})$ as a test function in (1.1) we have that

$$
\int_{\Omega}|\nabla u|^{n-2}\left\langle\nabla u, \varphi \nabla \chi_{\epsilon}+\chi_{\epsilon} \nabla \varphi\right\rangle=\int_{\Omega} \chi_{\epsilon}|x|^{n \alpha} e^{u} \varphi .
$$

Since $u \in W^{1, n-1}(\Omega)$ and $|x|^{n \alpha} e^{u} \in L^{1}(\Omega)$ it is easily seen that

$$
\begin{gathered}
\int_{\Omega} \chi_{\epsilon}|\nabla u|^{n-2}\langle\nabla u, \nabla \varphi\rangle \rightarrow \int_{\Omega}|\nabla u|^{n-2}\langle\nabla u, \nabla \varphi\rangle, \\
\int_{\Omega} \chi_{\epsilon}|x|^{n \alpha} e^{u} \varphi \rightarrow \int_{\Omega}|x|^{n \alpha} e^{u} \varphi
\end{gathered}
$$

as $\epsilon \rightarrow 0$. Since

$$
\int_{\Omega}|\nabla u|^{n-1}|\varphi-\varphi(0)|\left|\nabla \chi_{\epsilon}\right| \leq C \int_{B_{\epsilon}(0) \backslash B_{\frac{\epsilon}{2}}(0)}|\nabla u|^{n-1} \rightarrow 0
$$


as $\epsilon \rightarrow 0$ in view of $|\varphi-\varphi(0)| \leq C \epsilon$ in $B_{\epsilon}(0) \backslash B_{\frac{\epsilon}{2}}(0)$ and $u \in W^{1, n-1}(\Omega)$, the remaining term in (2.17) can be re-written as follows:

$$
\int_{\Omega}|\nabla u|^{n-2} \varphi\left\langle\nabla u, \nabla \chi_{\epsilon}\right\rangle=\varphi(0) \int_{\Omega}|\nabla u|^{n-2}\left\langle\nabla u, \nabla \chi_{\epsilon}\right\rangle+o(1)
$$

as $\epsilon \rightarrow 0$. By inserting (2.18)-(2.19) into (2.17) we deduce the existence of

$$
\gamma=\lim _{\epsilon \rightarrow 0} \int_{\Omega}|\nabla u|^{n-2}\left\langle\nabla u, \nabla \chi_{\epsilon}\right\rangle
$$

and the validity of (2.16) for $u$. Moreover, if we assume $u \in C^{1}(\bar{\Omega} \backslash\{0\})$, we can interpret $\gamma$ as

$$
\gamma=\lim _{\epsilon \rightarrow 0}\left[\int_{\Omega}|x|^{n \alpha} e^{u} \chi_{\epsilon}+\int_{\partial \Omega}|\nabla u|^{n-2} \partial_{n} u\right]=\int_{\Omega}|x|^{n \alpha} e^{u}+\int_{\partial \Omega}|\nabla u|^{n-2} \partial_{n} u .
$$

Since $\gamma_{r} \geq \gamma+o(1)$ as $r \rightarrow 0$ according to (4.16)- [11], we find that $h_{r}$ is possibly much lower than $u$ and then needs to be compensated by an unbounded function $u_{r} \geq 0$ in order to keep the validity of $u=u_{r}+h_{r}$. Instead, thanks to Theorem 2.1- [13] introduce $h \in \bigcap_{1 \leq q<n} W^{1, q}(B)$ as the solution of

$$
\Delta_{n} h=\gamma \delta_{0} \text { in } B, \quad h=u \text { on } \partial B
$$

so that

$$
h-\gamma\left(n \omega_{n}|\gamma|^{n-2}\right)^{-\frac{1}{n-1}} \log |x| \in L^{\infty}(B) .
$$

Decomposing $u$ as $u=u_{0}+h$, the solution $u_{0}$ of (1.16) on $B$ is very likely a bounded function, as we will prove below.

In order to establish some crucial integral inequalities involving $u_{0}$, let us introduce the following approximation scheme. By convolution with mollifiers consider sequences $f_{j}, g_{j} \in$ $C_{0}^{\infty}(B)$ so that $f_{j} \rightarrow|x|^{n \alpha} e^{u}-\gamma \delta_{0}$ weakly in the sense of measures and $0 \leq f_{j}-g_{j} \rightarrow$ $|x|^{n \alpha} e^{u}$ in $L^{1}(B)$ as $j \rightarrow+\infty$. Since $u \in C^{1, \eta}(\partial B)$, let $\varphi \in C^{1, \eta}(B)$ be the $n$-harmonic extension of $\left.u\right|_{\partial B}$ in $B$. Let $v_{j}, w_{j} \in W_{0}^{1, n}(B)$ be the weak solutions of $-\operatorname{div} \mathbf{a}\left(x, \nabla v_{j}\right)=f_{j}$ and $-\operatorname{div} \mathbf{a}\left(x, \nabla w_{j}\right)=g_{j}$ in $B$, where $\mathbf{a}(x, p)=|p+\nabla \varphi|^{n-2}(p+\nabla \varphi)-|\nabla \varphi|^{n-2} \nabla \varphi$. In this way, $u_{j}=v_{j}+\varphi$ and $h_{j}=w_{j}+\varphi$ do solve

$$
-\Delta_{n} u_{j}=f_{j} \text { and }-\Delta_{n} h_{j}=g_{j} \text { in } B, u_{j}=h_{j}=u \text { on } \partial B .
$$

Since $f_{j}, g_{j}$ are uniformly bounded in $L^{1}(B)$, by (21) in [4] we can assume that $v_{j} \rightarrow v$ and $w_{j} \rightarrow w$ in $W_{0}^{1, q}(B)$ for all $1 \leq q<n$ as $j \rightarrow+\infty$, where $v$ and $w$ do satisfy

$$
-\operatorname{div} \mathbf{a}(x, \nabla v)=|x|^{n \alpha} e^{u}-\gamma \delta_{0} \text { and }-\operatorname{div} \mathbf{a}(x, \nabla w)=-\gamma \delta_{0} \text { in } B
$$

in view of $g_{j} \rightarrow-\gamma \delta_{0}$ weakly in the sense of measures as $j \rightarrow+\infty$. Since $u-\varphi, h-\varphi \in$ $\bigcap W_{0}^{1, q}(B)$ do solve the first and the second equation in (2.21), respectively, by the $1 \leq q<n$

uniqueness result in [10] (see Theorems 1.2 and 4.2 in [10]) we have that $v=u-\varphi$ and $w=h-\varphi$, i.e.

$$
u_{j} \rightarrow u \text { and } h_{j} \rightarrow h \text { in } W^{1, q}(B) \text { for all } 1 \leq q<n \text { as } j \rightarrow+\infty .
$$

Thanks to the approximation given by the $u_{j}$ 's and $h_{j}$ 's, we can now derive some crucial integral inequalities on $u_{0}$. 
Proposition 2.3 Let $u_{0}$ be a solution of (1.16). Then $u_{0} \geq 0$ and we have:

$$
\int_{\left\{k<\left|u_{0}\right|<k+a\right\}}\left|\nabla u_{0}\right|^{n} \leq \frac{a}{d} \int_{B}|x|^{n \alpha} e^{u} \quad \forall k, a>0
$$

and, if $|x|^{n \alpha} e^{u} \in L^{p}(B)$ for some $p>1$,

$$
\left(\int_{B} u_{0}^{2 m n q}\right)^{\frac{1}{2 m}} \leq \frac{C q^{n-1}}{d}|B|^{\frac{n-1}{m n q}}\left(\int_{B}|x|^{n p \alpha} e^{p u}\right)^{\frac{1}{p}}\left(\int_{B} u_{0}^{m n q}\right)^{\frac{n(q-1)+1}{m n q}}
$$

where $m=\frac{p}{p-1}$ and

$$
d=\inf _{X \neq Y} \frac{\left\langle|X|^{n-2} X-|Y|^{n-2} Y, X-Y\right\rangle}{|X-Y|^{n}}>0
$$

Proof First use $-\left(v_{j}-w_{j}\right)_{-} \in W_{0}^{1, n}(B)$ as a test function for $-\operatorname{div} \mathbf{a}\left(x, \nabla v_{j}\right)+$ $\operatorname{div} \mathbf{a}\left(x, \nabla w_{j}\right)$ to get

$$
\begin{aligned}
d \int_{\left\{v_{j}-w_{j}<0\right\}}\left|\nabla\left(v_{j}-w_{j}\right)\right|^{n} & \leq-\int_{B}\left\langle\mathbf{a}\left(x, \nabla v_{j}\right)-\mathbf{a}\left(x, \nabla w_{j}\right), \nabla\left(v_{j}-w_{j}\right)_{-}\right\rangle \\
& =-\int_{B}\left(f_{j}-g_{j}\right)\left(v_{j}-w_{j}\right)_{-} \leq 0
\end{aligned}
$$

in view of (2.24) and $f_{j}-g_{j} \geq 0$. Hence, $v_{j}-w_{j} \geq 0$ and then $u_{0} \geq 0$ in view of $v_{j}-w_{j} \rightarrow u-h=u_{0}$ in $W_{0}^{1, q}(B)$ for all $1 \leq q<n$ as $j \rightarrow+\infty$. Now, introduce the truncature operator $T_{k, a}$, for $k, a>0$, as

$$
T_{k, a}(s)=\left\{\begin{array}{cl}
s-k \operatorname{sign}(s) & \text { if } k<|s|<k+a \\
a \operatorname{sign}(s) & \text { if }|s| \geq k+a \\
0 & \text { if }|s| \leq k
\end{array}\right.
$$

and use $T_{k, a}\left(v_{j}-w_{j}\right) \in W_{0}^{1, n}(B)$ as a test function for $-\operatorname{div} \mathbf{a}\left(x, \nabla v_{j}\right)+\operatorname{div} \mathbf{a}\left(x, \nabla w_{j}\right)$ to get

$$
\begin{aligned}
& d \int_{\left\{k<\left|v_{j}-w_{j}\right|<k+a\right\}}\left|\nabla\left(v_{j}-w_{j}\right)\right|^{n} \leq \int_{B}\left\langle\mathbf{a}\left(x, \nabla v_{j}\right)-\mathbf{a}\left(x, \nabla w_{j}\right), \nabla T_{k, a}\left(v_{j}-w_{j}\right)\right\rangle \\
& \quad=\int_{B}\left(f_{j}-g_{j}\right) T_{k, a}\left(v_{j}-w_{j}\right)
\end{aligned}
$$

in view of (2.24). Since $v_{j}-w_{j} \rightarrow u_{0}$ in $W_{0}^{1, q}(B)$ for all $1 \leq q<n$ and $f_{j}-g_{j} \rightarrow|x|^{n \alpha} e^{u}$ in $L^{1}(B)$ as $j \rightarrow+\infty$, we can let $j \rightarrow+\infty$ in (2.25) and get by Fatou's Lemma that

$$
d \int_{\left\{k<\left|u_{0}\right|<k+a\right\}}\left|\nabla u_{0}\right|^{n} \leq \int_{B}|x|^{n \alpha} e^{u} T_{k, a}\left(u_{0}\right) \leq a \int_{B}|x|^{n \alpha} e^{u}
$$

yielding the validity of (2.22). Finally, if $|x|^{n \alpha} e^{u} \in L^{p}(B)$ for some $p>1$, we can assume that $f_{j}-g_{j} \rightarrow|x|^{n \alpha} e^{u}$ in $L^{p}(B)$ as $j \rightarrow+\infty$ and use $T_{a}\left[\left|v_{j}-w_{j}\right|^{n(q-1)}\left(v_{j}-w_{j}\right)\right] \in$ $W_{0}^{1, n}(B)$, where $T_{a}=T_{0, a}$ and $a>0, q \geq 1$, as a test function for $-\operatorname{div} \mathbf{a}\left(x, \nabla v_{j}\right)+$ 
$\operatorname{div} \mathbf{a}\left(x, \nabla w_{j}\right)$ to get by Hölder's inequality

$$
\begin{aligned}
& d \frac{n(q-1)+1}{q^{n}} \int_{\left\{\left|v_{j}-w_{j}\right|^{n(q-1)+1}<a\right\}}|\nabla| v_{j}-\left.\left.w_{j}\right|^{q}\right|^{n} \\
& \leq \int_{B}\left|f_{j}-g_{j}\right|\left|v_{j}-w_{j}\right|^{n(q-1)+1} \\
& \leq|B|^{\frac{n-1}{m n q}}\left\|f_{j}-g_{j}\right\|_{p}\left(\int_{B}\left|v_{j}-w_{j}\right|^{m n q}\right)^{\frac{n(q-1)+1}{m n q}}
\end{aligned}
$$

in view of $\left|T_{a}(s)\right| \leq|s|$ and (2.24). We have used that $v_{j}-w_{j} \in W_{0}^{1, n}(B) \subset \bigcap_{q \geq 1} L^{q}(B)$ by the Sobolev embedding Theorem. Letting $a \rightarrow+\infty$, by Fatou's Lemma we get that

$$
\int_{B}|\nabla| v_{j}-\left.\left.w_{j}\right|^{q}\right|^{n} \leq \frac{q^{n}}{d[n(q-1)+1]}|B|^{\frac{n-1}{m n q}}\left\|f_{j}-g_{j}\right\|_{p}\left(\int_{B}\left|v_{j}-w_{j}\right|^{m n q}\right)^{\frac{n(q-1)+1}{m n q}} .
$$

In particular, $\left|v_{j}-w_{j}\right|^{q} \in W_{0}^{1, n}(B)$ and by the Sobolev embedding $W_{0}^{1, n}(B) \subset L^{2 m n}(B)$ we have that

$$
\left(\int_{B}\left|v_{j}-w_{j}\right|^{2 m n q}\right)^{\frac{1}{2 m}} \leq \frac{C q^{n}}{d[n(q-1)+1]}|B|^{\frac{n-1}{m n q}}\left\|f_{j}-g_{j}\right\|_{p}\left(\int_{B}\left|v_{j}-w_{j}\right|^{m n q}\right)^{\frac{n(q-1)+1}{m n q}} .
$$

Letting $j \rightarrow+\infty$, we finally deduce the validity of (2.23):

$$
\left(\int_{B} u_{0}^{2 m n q}\right)^{\frac{1}{2 m}} \leq \frac{C q^{n-1}}{d}|B|^{\frac{n-1}{m n q}}\left(\int_{B}|x|^{n p \alpha} e^{p u}\right)^{\frac{1}{p}}\left(\int_{B} u_{0}^{m n q}\right)^{\frac{n(q-1)+1}{m n q}}
$$

in view of $v_{j}-w_{j} \rightarrow u_{0}$ in $W_{0}^{1, q}(B)$ for all $1 \leq q<n$ and $f_{j}-g_{j} \rightarrow|x|^{n \alpha} e^{u}$ in $L^{p}(B)$ as $j \rightarrow+\infty$.

We are now ready to complete the proof of Theorem 1.1 .

Proof (of Theorem 1.1) Since $u_{0} \geq 0$ by Proposition 2.3, we have that $h \leq u$. By (1.1) and (2.20) we have that

$$
\int_{B}|x|^{n \alpha+\gamma\left(n \omega_{n}|\gamma|^{n-2}\right)^{-\frac{1}{n-1}}} \leq C \int_{B}|x|^{n \alpha} e^{h} \leq C \int_{\Omega}|x|^{n \alpha} e^{u}<+\infty,
$$

which implies

$$
n \alpha+\gamma\left(n \omega_{n}|\gamma|^{n-2}\right)^{-\frac{1}{n-1}}>-n
$$

or equivalently

$$
\gamma>-n^{n}|\alpha+1|^{n-2}(\alpha+1) \omega_{n} .
$$

Since $|x|^{n \alpha} e^{h} \in L^{1}$ near 0 and $h$ has a logarithmic singularity at 0 , then, as already observed in the Introduction, a stronger integrability follows:

$$
|x|^{n \alpha} e^{h} \in L^{p}(B)
$$

for some $p>1$. Inequality (2.22) is used in [1] to deduce exponential estimates on $u_{0}$ like

$$
\int_{B} e^{\frac{\delta u_{0}}{\|f\|_{1}}} \leq C_{r}
$$


for some $\delta>0$ where $f=|x|^{n \alpha} e^{u}$. Since $\lim _{r \rightarrow 0} \int_{B}|x|^{n \alpha} e^{u}=0$, by (2.29) we deduce that $e^{u_{0}} \in L^{p}(B)$ for all $p \geq 1$ if $r$ is sufficiently small and then by (2.28)

$$
|x|^{n \alpha} e^{u}=|x|^{n \alpha} e^{h} e^{u_{0}} \in L^{p}(B)
$$

for some $p>1$. Inequality (2.23) is used in Proposition 4.1-[11] (compare with (4.4) in [11]) to get $u_{0} \in L^{\infty}(B)$ and then (2.20) does hold for $u$ too, yielding the validity of (1.3). In order to prove (1.4), set $H=u-\gamma\left(n \omega_{n}|\gamma|^{n-2}\right)^{-\frac{1}{n-1}} \log |x|$ and introduce the function

$$
U_{r}(y)=u(r y)-\gamma\left(n \omega_{n}|\gamma|^{n-2}\right)^{-\frac{1}{n-1}} \log r=\gamma\left(n \omega_{n}|\gamma|^{n-2}\right)^{-\frac{1}{n-1}} \log |y|+H(r y)
$$

for a given sequence $r \rightarrow 0$. Since

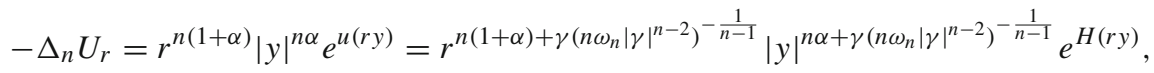

by (1.3) and (2.26)-(2.27) we have that $U_{r}$ and $\Delta_{n} U_{r}$ are bounded in $L_{\text {loc }}^{\infty}\left(\mathbb{R}^{n} \backslash\{0\}\right)$, uniformly in $r$. By $[9,19,22]$ we deduce that $U_{r}$ is bounded in $C_{\text {loc }}^{1, \eta}\left(\mathbb{R}^{n} \backslash\{0\}\right)$, uniformly in $r$. By the Ascoli-Arzelá's Theorem and a diagonal process, up to a sub-sequence we have that $U_{r} \rightarrow U_{0}$ in $C_{\text {loc }}^{1}\left(\mathbb{R}^{n} \backslash\{0\}\right)$, where $U_{0}$ is a n-harmonic function in $\mathbb{R}^{n} \backslash\{0\}$. Setting $H_{r}(y)=H(r y)$, we deduce that $H_{r} \rightarrow H_{0}$ in $C_{\text {loc }}^{1}\left(\mathbb{R}^{n} \backslash\{0\}\right)$, where $H_{0} \in L^{\infty}\left(\mathbb{R}^{n}\right)$ in view of (1.3). Since $U_{0}=\gamma\left(n \omega_{n}|\gamma|^{n-2}\right)^{-\frac{1}{n-1}} \log |y|+H_{0}$ with $H_{0} \in L^{\infty}\left(\mathbb{R}^{n}\right) \cap C^{1}\left(\mathbb{R}^{n} \backslash\{0\}\right)$, it is well known that $H_{0}$ is a constant function, as shown in Corollary 2.2-[13] (see also [11] for a direct proof). In particular we get that

$$
\sup _{|x|=r}|x|\left|\nabla\left[u-\gamma\left(n \omega_{n}|\gamma|^{n-2}\right)^{-\frac{1}{n-1}} \log |x|\right]\right|=\sup _{|y|=1}\left|\nabla H_{r}\right| \rightarrow \sup _{|y|=1}\left|\nabla H_{0}\right|=0 .
$$

Since this is true for any sequence $r \rightarrow 0$ up to extracting a sub-sequence, we have established the validity of (1.4). The proof of Theorem 1.1 is concluded.

\section{Quantization results}

In this section we will make crucial use of the following integral identity: for any solution $u$ of

$$
-\Delta_{n} u=|x|^{n \alpha} e^{u} \text { in } \mathbb{R}^{n} \backslash\{0\}
$$

there holds

$$
n(\alpha+1) \int_{A}|x|^{n \alpha} e^{u}=\int_{\partial A}\left[|x|^{n \alpha} e^{u}\langle x, \nu\rangle+|\nabla u|^{n-2} \partial_{\nu} u\langle\nabla u, x\rangle-\frac{|\nabla u|^{n}}{n}\langle x, \nu\rangle\right],
$$

where $A$ is the annulus $A=B_{R}(0) \backslash B_{\epsilon}(0), 0<\epsilon<R<+\infty$, and $v$ is the unit outward normal vector at $\partial A$. Notice that (3.2) is simply a special case of the well-known Pohozaev identities associated to (3.1). Even though the classical Pohozaev identities require more regularity than simply $u \in C^{1, \eta}\left(\mathbb{R}^{n} \backslash\{0\}\right),(3.2)$ is still valid in the quasilinear case and we refer to [8] for a justification. Thanks to (3.2) we are able to show the following general result. 
Proposition 3.1 Let $u$ be a solution of (3.1) so that (1.3)-(1.4) do hold at 0 and $\infty$ with $\gamma$ and $-\gamma_{\infty}$, respectively, so that $\gamma>-n^{n}|\alpha+1|^{n-2}(\alpha+1) \omega_{n}$ and $\gamma_{\infty}>n^{n}|\alpha+1|^{n-2}(\alpha+1) \omega_{n}$. Then $\int_{\mathbb{R}^{n}}|x|^{n \alpha} e^{u}=\gamma+\gamma_{\infty}$ satisfies

$$
n(\alpha+1)\left(\gamma+\gamma_{\infty}\right)=\frac{n-1}{n}\left(n \omega_{n}\right)^{-\frac{1}{n-1}}\left[\left|\gamma_{\infty}\right|^{\frac{n}{n-1}}-|\gamma|^{\frac{n}{n-1}}\right] .
$$

Proof By (1.3)-(1.4) at 0 with $\gamma>-n^{n}|\alpha+1|^{n-2}(\alpha+1) \omega_{n}$ we deduce that

$$
\begin{aligned}
& |\nabla u|=\frac{1}{|x|}\left[\left(\frac{|\gamma|}{n \omega_{n}}\right)^{\frac{1}{n-1}}+o(1)\right], \quad\langle\nabla u, x\rangle=\gamma\left(n \omega_{n}|\gamma|^{n-2}\right)^{-\frac{1}{n-1}}+o(1), \\
& |x|^{n \alpha} e^{u}=o\left(\frac{1}{|x|^{n}}\right)
\end{aligned}
$$

as $x \rightarrow 0$ thanks to the equivalence between (2.26) and (2.27). By (3.4) we have that

$$
\int_{\partial B_{\epsilon}(0)}|x|\left[|x|^{n \alpha} e^{u}+|\nabla u|^{n-2}\left\langle\nabla u, \frac{x}{|x|}\right\rangle^{2}-\frac{|\nabla u|^{n}}{n}\right] \rightarrow \frac{n-1}{n}\left(n \omega_{n}\right)^{-\frac{1}{n-1}}|\gamma|^{\frac{n}{n-1}}
$$

as $\epsilon \rightarrow 0^{+}$in view of Area $\left(\mathbb{S}^{n-1}\right)=n \omega_{n}$. Similarly, by (1.3)-(1.4) at $\infty$ with $-\gamma_{\infty}$ so that $\gamma_{\infty}>n^{n}|\alpha+1|^{n-2}(\alpha+1) \omega_{n}$ we deduce that

$$
\begin{aligned}
& |\nabla u|=\frac{1}{|x|}\left[\left(\frac{\left|\gamma_{\infty}\right|}{n \omega_{n}}\right)^{\frac{1}{n-1}}+o(1)\right], \quad\langle\nabla u, x\rangle=-\gamma_{\infty}\left(n \omega_{n}\left|\gamma_{\infty}\right|^{n-2}\right)^{-\frac{1}{n-1}}+o(1), \\
& |x|^{n \alpha} e^{u}=o\left(\frac{1}{|x|^{n}}\right)
\end{aligned}
$$

as $|x| \rightarrow \infty$ and then

$$
\int_{\partial B_{R}(0)}|x|\left[|x|^{n \alpha} e^{u}+|\nabla u|^{n-2}\left\langle\nabla u, \frac{x}{|x|}\right\rangle^{2}-\frac{|\nabla u|^{n}}{n}\right] \rightarrow \frac{n-1}{n}\left(n \omega_{n}\right)^{-\frac{1}{n-1}}\left|\gamma_{\infty}\right|^{\frac{n}{n-1}}
$$

as $R \rightarrow+\infty$. In view of (1.4) at 0 and $\infty$ we easily get that

$$
-\Delta_{n} u=|x|^{n \alpha} e^{u}-\gamma \delta_{0}-\gamma_{\infty} \delta_{\infty} \text { in } \mathbb{R}^{n}
$$

in the sense

$$
\int_{\mathbb{R}^{n}}|\nabla u|^{n-2}\langle\nabla u, \nabla \varphi\rangle=\int_{\mathbb{R}^{n}}|x|^{n \alpha} e^{u} \varphi-\gamma \varphi(0)-\gamma_{\infty} \varphi(\infty)
$$

for all $\varphi \in C^{1}\left(\mathbb{R}^{n}\right)$ so that $\varphi(\infty):=\lim _{|x| \rightarrow \infty} \varphi(x)$ does exist. Choosing $\varphi=1$ we deduce that

$$
\int_{\mathbb{R}^{n}}|x|^{n \alpha} e^{u}=\gamma+\gamma_{\infty}
$$

By inserting (3.5)-(3.7) into (3.2) and letting $\epsilon \rightarrow 0^{+}, R \rightarrow+\infty$ we deduce the validity of (3.3).

Let us now apply Proposition 3.1 to problems (1.12) and (1.15). 
Proof (of Theorem 1.3) Let $u$ be a solution of (1.12). By Theorem 1.1 (1.3)-(1.4) do hold for $u$ at 0 with $\gamma>-n^{n} \omega_{n}$. By (1.6) the Kelvin transform $\hat{u}$ satisfies

$$
-\Delta_{n} \hat{u}=|x|^{-2 n} e^{\hat{u}} \text { in } \mathbb{R}^{n} \backslash\{0\} .
$$

Let us apply Theorem 1.1 to deduce the validity of (1.3)-(1.4) for $\hat{u}$ at 0 with $\gamma_{\infty}>n^{n} \omega_{n}$. Back to $u$, (1.3)-(1.4) do hold for $u$ at $\infty$ with $-\gamma_{\infty}$ so that $\gamma_{\infty}>n^{n} \omega_{n}$. Let us apply Proposition 3.1 with $\alpha=0$ to get $\int_{\mathbb{R}^{n}} e^{u}=\gamma+\gamma_{\infty}$ with $\gamma_{\infty}$ satisfying (1.14). Notice that the function $f(s)=n s+\frac{n-1}{n}\left(n \omega_{n}\right)^{-\frac{1}{n-1}}|s|^{\frac{n}{n-1}}$ is increasing in $\left(-n^{n} \omega_{n},+\infty\right)$ and then $f(s)>f\left(-n^{n} \omega_{n}\right)=-n^{n} \omega_{n}$ for all $s \in\left(-n^{n} \omega_{n},+\infty\right)$. At the same time the function $g(s)=\frac{n-1}{n}\left(n \omega_{n}\right)^{-\frac{1}{n-1}} s^{\frac{n}{n-1}}-n s$ is increasing in $\left(n^{n} \omega_{n},+\infty\right)$ and then $g(s)>g\left(n^{n} \omega_{n}\right)=$ $-n^{n} \omega_{n}$ for all $s \in\left(n^{n} \omega_{n},+\infty\right)$. Therefore, for any $\gamma>-n^{n} \omega_{n}$ Equation (1.14) has a unique solution $\gamma_{\infty}>n^{n} \omega_{n}$. The proof of Theorem 1.3 is concluded.

Remark 3.2 Concerning Corollary 1.2, observe that in the argument above we have established (1.7) for problem (1.5) on $\Omega=\mathbb{R}^{n}$ and a similar proof is in order for a general unbounded open set $\Omega$. Since $\gamma=0$, we deduce the validity of (1.8) in view of (1.13).

Proof (of Theorem 1.4) Let $v$ be a solution of (1.15). Applying Theorem 1.1 to the Kelvin transform $\hat{v}$, solution of

$$
-\Delta_{n} \hat{v}=|x|^{-n(\alpha+2)} e^{\hat{v}} \text { in } \mathbb{R}^{n} \backslash\{0\},
$$

we deduce the validity of (1.3)-(1.4) for $v$ at $\infty$ with $-\gamma_{\infty}$ so that $\gamma_{\infty}>n^{n}|\alpha+1|^{n-2}(\alpha+$ 1) $\omega_{n}$. By Proposition 3.1 with $\gamma=0$ we deduce that $\gamma_{\infty}=\int_{\mathbb{R}^{n}}|x|^{n \alpha} e^{v}$ satisfies

$$
n(\alpha+1) \gamma_{\infty}=\frac{n-1}{n}\left(n \omega_{n}\right)^{-\frac{1}{n-1}} \gamma_{\infty}^{\frac{n}{n-1}}
$$

Therefore, $\alpha>-1$ and

$$
\int_{\mathbb{R}^{n}}|x|^{n \alpha} e^{v}=n\left(\frac{n^{2}}{n-1}\right)^{n-1}(\alpha+1)^{n-1} \omega_{n},
$$

concluding the proof of Theorem 1.4.

\section{Radial solutions for (1.12)}

Fix $M>1$ and assume that

$$
\frac{1}{M} \leq r_{0} \leq M, \quad \alpha_{0} \leq M, \quad \frac{1}{M} \leq\left|\alpha_{1}\right| \leq M .
$$

Let us first discuss the local existence theory for the following Cauchy problem:

$$
\left\{\begin{array}{c}
-\frac{1}{r^{n-1}}\left(r^{n-1}\left|U^{\prime}\right|^{n-2} U^{\prime}\right)^{\prime}=e^{U} \\
U\left(r_{0}\right)=\alpha_{0}, \quad U^{\prime}\left(r_{0}\right)=\alpha_{1}
\end{array}\right.
$$

Given $0<\delta<\frac{1}{2 M}$, define $I=\left[r_{0}-\delta, r_{0}+\delta\right]$ and $E=\left\{U \in C\left(I,\left[\alpha_{0}-1, \alpha_{0}+1\right]\right)\right.$ : $\left.U\left(r_{0}\right)=\alpha_{0}\right\}$, which is a Banach space endowed with $\|\cdot\|_{\infty}$ as a norm. We can re-formulate 
(4.2) as $U=T U$, where

$$
\begin{aligned}
T U(r)= & \alpha_{0}+\left.\int_{r_{0}}^{r} \frac{d s}{s}\left|r_{0}^{n-1}\right| \alpha_{1}\right|^{n-2} \alpha_{1} \\
& -\left.\int_{r_{0}}^{s} t^{n-1} e^{U(t)} d t\right|^{-\frac{n-2}{n-1}}\left(r_{0}^{n-1}\left|\alpha_{1}\right|^{n-2} \alpha_{1}-\int_{r_{0}}^{s} t^{n-1} e^{U(t)} d t\right) .
\end{aligned}
$$

In view of

$$
\left|s^{n}-r_{0}^{n}\right| \leq n(M+1)^{n-1} \delta \quad \forall s \in I
$$

we have that $\max _{I} U \leq M+1$ and $\max _{I}\left|\int_{r_{0}}^{s} t^{n-1} e^{U(t)} d t\right| \leq e^{M+1}(M+1)^{n-1} \delta$ for all $U \in E$, and then for $0<\delta<\frac{e^{-M-1}}{2(M+1)^{3 n-3}}$ we have that

$$
r_{0}^{n-1}\left|\alpha_{1}\right|^{n-2} \alpha_{1}-\int_{r_{0}}^{s} t^{n-1} e^{U(t)} d t \text { has the same sign as } \alpha_{1} \quad \forall s \in I
$$

and

$$
\begin{aligned}
\frac{1}{2 M^{2 n-2}} \leq & \frac{1}{2} r_{0}^{n-1}\left|\alpha_{1}\right|^{n-1} \leq\left.\left|r_{0}^{n-1}\right| \alpha_{1}\right|^{n-2} \alpha_{1} \\
& -\left.\int_{r_{0}}^{s} t^{n-1} e^{U(t)} d t\left|\leq \frac{3}{2} r_{0}^{n-1}\right| \alpha_{1}\right|^{n-1} \leq \frac{3}{2} M^{2 n-2}
\end{aligned}
$$

for all $U \in E$. Since $\log \frac{r_{0}+\delta}{r_{0}} \leq \log \frac{r_{0}}{r_{0}-\delta} \leq \frac{\delta}{r_{0}-\delta} \leq 2 M \delta$ in view of $\delta<\frac{r_{0}}{2}$ and

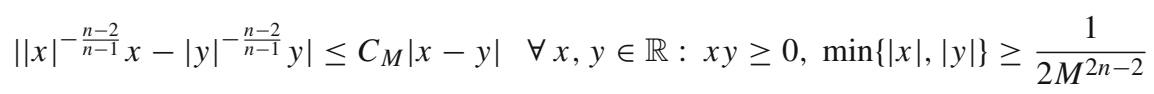

(for example, take $\left.C_{M}=\left(1+\frac{n-2}{n-1}\right)\left(4 M^{2 n-2}\right)^{\frac{n-2}{n-1}}\right)$, by (4.4)-(4.5) we have that

$$
\begin{aligned}
\left\|T U-\alpha_{0}\right\|_{\infty, I} & \leq \sup _{r \in I}\left|\int_{r_{0}}^{r} \frac{d s}{s}\right| r_{0}^{n-1}\left|\alpha_{1}\right|^{n-2} \alpha_{1}-\left.\int_{r_{0}}^{s} t^{n-1} e^{U(t)} d t\right|^{\frac{1}{n-1}} \mid \\
& \leq 2\left(\frac{3}{2}\right)^{\frac{1}{n-1}} M^{3} \delta \leq 3 M^{3} \delta
\end{aligned}
$$

and

$$
\begin{aligned}
\|T U-T V\|_{\infty, I} & \leq C_{M} \sup _{r \in I}\left|\int_{r_{0}}^{r} \frac{d s}{s}\right| \int_{r_{0}}^{s} t^{n-1}\left[e^{U(t)}-e^{V(t)}\right] d t \| \\
& \leq 2 C_{M}(M+1)^{n} e^{M+1} \delta\|U-V\|_{\infty, I}
\end{aligned}
$$

for all $U, V \in C^{1}(I)$ in view of $\delta<1$ and (4.3). In conclusion, if

$$
0<\delta<\min \left\{\frac{1}{3 M^{3}}, \frac{e^{-M-1}}{2(M+1)^{3 n-3}}, \frac{e^{-M-1}}{2 C_{M}(M+1)^{n}}\right\},
$$

then $T$ is a contraction map from $E$ into itself and a unique fixed point $U \in E$ of $T$ is found by the Contraction Mapping Theorem, providing a solution $U$ of (4.2) in $I=\left[r_{0}-\delta, r_{0}+\delta\right]$.

Once a local existence result has been established for (4.2), we can turn the attention to global issues. Given $r_{0}>0, \alpha_{0}$ and $\alpha_{1} \neq 0$, let $I=\left(r_{1}, r_{2}\right), 0 \leq r_{1}<r_{0}<r_{2} \leq+\infty$, be the maximal interval of existence for the solution $U$ of (4.2). We claim that $r_{1}=0$ when $\alpha_{1}>0$ and $r_{2}=+\infty$ when $\alpha_{1}<0$. 
Consider first the case $\alpha_{1}>0$ and assume by contradiction $r_{1}>0$. Since

$$
U^{\prime}(r)=\frac{1}{r}\left(r_{0}^{n-1} \alpha_{1}^{n-1}+\int_{r}^{r_{0}} t^{n-1} e^{U(t)} d t\right)^{\frac{1}{n-1}} \geq \frac{r_{0} \alpha_{1}}{r}>0
$$

for all $r \in\left(r_{1}, r_{0}\right]$, one would have that

$$
U(r) \leq \alpha_{0}, \quad \alpha_{1} \leq U^{\prime}(r) \leq \frac{1}{r_{1}}\left[r_{0}^{n-1} \alpha_{1}^{n-1}+\frac{r_{0}^{n}}{n} e^{\alpha_{0}}\right]^{\frac{1}{n-1}}
$$

for all $r \in\left(r_{1}, r_{0}\right]$ and then (4.1) would hold for initial conditions $\alpha_{0}^{\prime}=U\left(r_{0}^{\prime}\right), \alpha_{1}^{\prime}=U^{\prime}\left(r_{0}^{\prime}\right)$ in (4.2) at $r_{0}^{\prime}$ approaching $r_{1}$ from the right. Since this would allow to continue the solution $U$ on the left of $r_{1}$ in view of the estimate (4.6) on the time for local existence, we would reach a contradiction and then the property $r_{1}=0$ has been established.

In the case $\alpha_{1}<0$ assume by contradiction $r_{2}<+\infty$. Since

$$
U^{\prime}(r)=-\frac{1}{r}\left(r_{0}^{n-1}\left|\alpha_{1}\right|^{n-1}+\int_{r_{0}}^{r} t^{n-1} e^{U(t)} d t\right)^{\frac{1}{n-1}} \leq-\frac{r_{0}\left|\alpha_{1}\right|}{r}<0
$$

for all $r \in\left[r_{0}, r_{2}\right)$, one would have that

$$
U(r) \leq \alpha_{0}, \quad-\frac{1}{r_{0}}\left[r_{0}^{n-1}\left|\alpha_{1}\right|^{n-1}+\frac{r_{2}^{n}}{n} e^{\alpha_{0}}\right]^{\frac{1}{n-1}} \leq U^{\prime}(r) \leq-\frac{r_{0}\left|\alpha_{1}\right|}{r_{2}}
$$

for all $r \in\left[r_{0}, r_{2}\right)$ and then (4.1) would hold for initial conditions $\alpha_{0}^{\prime}=U\left(r_{0}^{\prime}\right), \alpha_{1}^{\prime}=U^{\prime}\left(r_{0}^{\prime}\right)$ in (4.2) at $r_{0}^{\prime}$ approaching $r_{2}$ from the left. Since one could continue the solution $U$ past $r_{2}$ thanks to (4.6), a contradiction would arise. Then, we have shown that $r_{2}=+\infty$.

Given $\epsilon>0$, let now $U_{\epsilon}^{ \pm}$be the maximal solution of

$$
\left\{\begin{array}{c}
-\frac{1}{r^{n-1}}\left(r^{n-1}\left|U^{\prime}\right|^{n-2} U^{\prime}\right)^{\prime}=e^{U} \\
U(1)=\alpha_{0}, \quad U^{\prime}(1)= \pm \epsilon
\end{array}\right.
$$

By the discussion above we have that $U_{\epsilon}^{+}$and $U_{\epsilon}^{-}$are well defined in $(0,1]$ and $[1,+\infty)$, respectively. According to (4.7)-(4.8) one has

$$
\begin{aligned}
& \left(U_{\epsilon}^{+}\right)^{\prime}=\frac{1}{r}\left(\epsilon^{n-1}+\int_{r}^{1} t^{n-1} e^{U_{\epsilon}^{+}(t)} d t\right)^{\frac{1}{n-1}} \text { in }(0,1], \\
& \left(U_{\epsilon}^{-}\right)^{\prime}=-\frac{1}{r}\left(\epsilon^{n-1}+\int_{1}^{r} t^{n-1} e^{U_{\epsilon}^{-}(t)} d t\right)^{\frac{1}{n-1}} \text { in }[1,+\infty)
\end{aligned}
$$

and then $U_{\epsilon}^{+}, U_{\epsilon}^{-}$are uniformly bounded in $C_{l o c}^{1, \gamma}(0,1], C_{l o c}^{1, \gamma}[1,+\infty)$, respectively, in view of $U_{\epsilon}^{+}, U_{\epsilon}^{-} \leq \alpha_{0}$. Up to a subsequence and a diagonal argument, we can assume that $U_{\epsilon}^{+} \rightarrow U^{+}$ in $C_{l o c}^{1}(0,1]$ and $U_{\epsilon}^{-} \rightarrow U^{-}$in $C_{l o c}^{1}[1,+\infty)$ as $\epsilon \rightarrow 0^{+}$, where

$$
\begin{aligned}
& \left(U^{+}\right)^{\prime}=\frac{1}{r}\left(\int_{r}^{1} t^{n-1} e^{U^{+}(t)} d t\right)^{\frac{1}{n-1}} \text { in }(0,1], \\
& \left(U^{-}\right)^{\prime}=-\frac{1}{r}\left(\int_{1}^{r} t^{n-1} e^{U_{-}(t)} d t\right)^{\frac{1}{n-1}} \text { in }[1,+\infty)
\end{aligned}
$$

thanks to (4.9). Since $U^{+}(1)=U^{-}(1)=\alpha_{0}$ and $\left(U^{+}\right)^{\prime}(1)=\left(U^{-}\right)^{\prime}(1)=0$ in view of (4.10), we have that

$$
U=\left\{\begin{array}{l}
U^{+} \text {in }(0,1] \\
U^{-} \text {in }[1,+\infty)
\end{array}\right.
$$


is in $C^{1}(0,+\infty)$ with $U \leq U(1)=\alpha_{0}, U^{\prime}(1)=0$ and

$$
U^{\prime}(r)=\frac{1}{r}\left|\int_{r}^{1} t^{n-1} e^{U(t)} d t\right|^{-\frac{n-2}{n-1}} \int_{r}^{1} t^{n-1} e^{U(t)} d t \text { in }(0,+\infty) .
$$

It is not difficult to check that $U$ satisfies $-\Delta_{n} U=e^{U}$ in $\mathbb{R}^{n} \backslash\{0\}$ and

$$
\lim _{r \rightarrow 0} \frac{U(r)}{\log r}=\lim _{r \rightarrow 0} r U^{\prime}(r)=\left(\int_{0}^{1} t^{n-1} e^{U(t)} d t\right)^{\frac{1}{n-1}}=\left(\frac{1}{n \omega_{n}} \int_{B_{1}(0)} e^{U}\right)^{\frac{1}{n-1}}
$$

in view of (4.11). By Theorem 1.1 and (4.12) we deduce that $U$ is a radial solution of

$$
-\Delta_{n} U=e^{U}-\gamma \delta_{0} \text { in } \mathbb{R}^{n}, \quad U \leq U(1)=\alpha_{0},
$$

with $\gamma=\int_{B_{1}(0)} e^{U}$ depending on the choice of $\alpha_{0}$. By the Pohozaev identity (3.2) on $A=B_{1}(0) \backslash B_{\epsilon}(0), \epsilon \in(0,1)$, we have that

$$
\omega_{n}\left[e^{\alpha_{0}}-\epsilon^{n} e^{U(\epsilon)}\right]=\int_{B_{1}(0) \backslash B_{\epsilon}(0)} e^{U}+\frac{n-1}{n} \omega_{n}\left[\epsilon U^{\prime}(\epsilon)\right]^{n}
$$

in view of $U(1)=\alpha_{0}$ and $U^{\prime}(1)=0$, and letting $\epsilon \rightarrow 0^{+}$one deduces that

$$
\omega_{n} e^{\alpha_{0}}=\gamma+\frac{n-1}{n} \omega_{n}\left(\frac{\gamma}{n \omega_{n}}\right)^{\frac{n}{n-1}}
$$

in view of (4.12). Since $\gamma \in(0,+\infty) \rightarrow \gamma+\frac{n-1}{n} \omega_{n}\left(\frac{\gamma}{n \omega_{n}}\right)^{\frac{n}{n-1}} \in(0,+\infty)$ is a bijection, for any given $\gamma>0$ let $\alpha_{0}=\log \left[\frac{\gamma}{\omega_{n}}+\frac{n-1}{n}\left(\frac{\gamma}{n \omega_{n}}\right)^{\frac{n}{n-1}}\right]$ and the corresponding $U$ is the solution of (1.12) we were searching for. Notice that $\int_{\mathbb{R}^{n}} e^{U}<+\infty$ in view of $\int_{1}^{\infty} t^{n-1} e^{U(t)} d t<+\infty$, as it can be deduced by

$$
\lim _{r \rightarrow+\infty} \frac{U(r)}{\log r}=\lim _{r \rightarrow+\infty} r U^{\prime}(r)=-\left(\int_{1}^{\infty} t^{n-1} e^{U(t)} d t\right)^{\frac{1}{n-1}}
$$

due to (4.11). We have established the following result:

Theorem 4.1 For any $\gamma>0$ there exists a 1-parameter family of distinct solutions $U_{\lambda}, \lambda>0$, to (1.12) given by $U_{\lambda}(x)=U(\lambda x)+n \log \lambda$ such that $U_{\lambda}$ takes its unique absolute maximum point at $\frac{1}{\lambda}$.

Acknowledgements The author would like to thank the referee for a careful reading and for pointing out a mistake in the original argument.

Funding Open access funding provided by Università degli Studi Roma Tre within the CRUI-CARE Agreement.

Open Access This article is licensed under a Creative Commons Attribution 4.0 International License, which permits use, sharing, adaptation, distribution and reproduction in any medium or format, as long as you give appropriate credit to the original author(s) and the source, provide a link to the Creative Commons licence, and indicate if changes were made. The images or other third party material in this article are included in the article's Creative Commons licence, unless indicated otherwise in a credit line to the material. If material is not included in the article's Creative Commons licence and your intended use is not permitted by statutory regulation or exceeds the permitted use, you will need to obtain permission directly from the copyright holder. To view a copy of this licence, visit http://creativecommons.org/licenses/by/4.0/. 


\section{References}

1. Aguilar Crespo, J.A., Peral Alonso, I.: Blow-up behavior for solutions of $-\Delta_{N} u=V(x) e^{u}$ in bounded domains in $\mathbb{R}^{N}$. Nonlinear Anal. 29(4), 365-384 (1997)

2. Bartolucci, D., Tarantello, G.: Liouville type equations with singular data and their applications to periodic multivortices for the electroweak theory. Commun. Math. Phys. 229(1), 3-47 (2002)

3. Bénilan, P., Boccardo, L., Gallouët, T., Gariepy, R., Pierre, M., Vázquez, J.L.: An $L^{1}$-theory of existence and uniqueness of solutions of nonlinear elliptic equations. Ann. Scuola Norm. Sup. Pisa Cl. Sci. (4) 22(2), 241-273 (1995)

4. Boccardo, L., Gallouët, T.: Nonlinear elliptic and parabolic equations involving measure data. J. Funct. Anal. 87(1), 149-169 (1989)

5. Brézis, H., Merle, F.: Uniform estimates and blow-up behavior for solutions of $-\Delta u=V(x) e^{u}$ in two dimensions. Commun. Partial Differ. Equ. 16(8-9), 1223-1253 (1991)

6. Chen, W., Li, C.: Classification of solutions of some nonlinear elliptic equations. Duke Math. J. 63(3), 615-623 (1991)

7. Chou, K.S., Wan, T.Y.H.: Asymptotic radial symmetry for solutions of $\Delta u+e^{u}=0$ in a punctured disc. Pac. J. Math. 163, 269-276 (1994)

8. Damascelli, L., Farina, A., Sciunzi, B., Valdinoci, E.: Liouville results for m-Laplace equations of LameEmden-Fowler type. Ann. Inst. H. Poincaré Anal. Non Linéaire 26(4), 1099-1119 (2009)

9. DiBenedetto, E.: $C^{1+\alpha}$ local regularity of weak solutions of degenerate elliptic equations. Nonlinear Anal. 7(8), 827-850 (1983)

10. Dolzmann, G., Hungerbühler, N., Müller, S.: Uniqueness and maximal regularity for nonlinear elliptic systems of n-Laplace type with measure valued right hand side. J. Reine Angew. Math. 520, 1-35 (2000)

11. Esposito, P.: A classification result for the quasi-linear Liouville equation. Ann. Inst. H. Poincaré Anal. Non Linéaire 35(3), 781-801 (2018)

12. Esposito, P., Morlando, F.: On a quasilinear mean field equation with an exponential nonlinearity. J. Math. Pures Appl. (9) 104(2), 354-382 (2015)

13. Kichenassamy, S., Veron, L.: Singular solutions of the $p$-Laplace equation. Math. Ann. 275(4), 599-615 (1986)

14. Li, Y.Y., Shafrir, I.: Blow-up analysis for solutions of $-\Delta u=V e^{u}$ in dimension two. Indiana Univ. Math. J. 43(4), 1255-1270 (1994)

15. Lieberman, G.M.: Boundary regularity for solutions of degenerate elliptic equations. Nonlinear Anal. 12(11), 1203-1219 (1988)

16. Liouville, J.: Sur l'équation aud dérivées partielles $\partial^{2} \log \lambda / \partial u \partial v \pm 2 \lambda a^{2}=0$. J. de Math. 18, 71-72 (1853)

17. Prajapat, J., Tarantello, G.: On a class of elliptic problems in $\mathbb{R}^{2}$ : symmetry and uniqueness results. Proc. R. Soc. Edinb. 131(4), 967-985 (2001)

18. Robert, F., Wei, J.: Asymptotic behavior of a fourth order mean field equation with Dirichlet boundary condition. Indiana Univ. Math. J. 57(5), 2039-2060 (2008)

19. Serrin, J.: Local behavior of solutions of quasilinear equations. Acta Math. 111, 247-302 (1964)

20. Serrin, J.: Isolated singularities of solutions of quasi-linear equations. Acta Math. 113, 219-240 (1965)

21. Tarantello, G.: A quantization property for blow-up solutions of singular Liouville-type equations. J. Funct. Anal. 219, 368-399 (2005)

22. Tolksdorf, P.: Regularity for more general class of quasilinear elliptic equations. J. Differ. Equ. 51(1), 126-150 (1984)

Publisher's Note Springer Nature remains neutral with regard to jurisdictional claims in published maps and institutional affiliations. 